\title{
Measurement Procedure for Surface Emissivity of Heat-Shielding Materials
}

\author{
Vladimir Arkhipov ${ }^{1,2}$, Irina Zharova ${ }^{1, \mathrm{a}}$, Viktor Gol'din ${ }^{1}$, and Natalya Korovina ${ }^{2}$ \\ ${ }^{1}$ National Research Tomsk State University, 634050 Tomsk, Russia \\ ${ }^{2}$ Institute for Problems of Chemical and Energetic Technologies, 659322 Biysk, Russia
}

\begin{abstract}
A procedure is suggested for the measurement of the integral emissivity coefficient of heat-shielding materials in the temperature range close to the thermal destruction temperature.
\end{abstract}

\section{Introduction}

The relevance of experimental studies into characteristics of heat-barrier materials is governed by the necessity of using the same in mechanical engineering, construction industry, power engineering, etc. Of most importance is the knowledge about behaviors of heat-shielding materials applied in thermal protection systems of rocket and space hardware while examining thermophysical properties of the materials used as heat barriers and insulators in flow passages of rocket propulsions and the like [1].

One of the basic features of heat exchange is the emissivity coefficient of solid bodies. In the general case, the emissivity coefficient of heat-shielding materials is dependent on many parameters such as properties of the substance itself, surface state and shape of the body under question, temperature, wavelength or wavelength range, direction of emission propagation, etc.

To measure the integral emissivity coefficient of the surface of various materials over a wide temperature range, a number of methods and corresponding designs of experimental instruments are currently in use. A quite complete review on this issue can be found in the monograph [2]. The radiation, calorimetric, and unsteady-state methods have become widely used in the practice of laboratory studies.

The radiation method determines emissivity coefficient using a dedicated thermal detector to comparatively measure the radiant energy emitted by a body under examination or by absolutely black body or by a body whose emissivity coefficient is unknown. In the range between room temperature and melting point or destruction temperature of a material under study, the calorimetric method has the most common use. In contrast to the radiation method, the calorimetric one relies on directly measuring the amount of energy emitted by a body. Unsteady-state methods are applied to measuring the integral emissivity coefficient over a wide temperature range, from helium temperatures to about $1200 \mathrm{~K}$. The unsteadystate measurement methods are premised on using the heat balance equation when a specimen under study is cooled by radiation in vacuum; the equation is written under the assumption that the temperature is uniformly distributed in the specimen volume. The

\footnotetext{
${ }^{\text {a }}$ Corresponding author: zharova@niipmm.tsu.ru
} 
advantage of the unsteady-state methods is that studies can be performed in different gaseous media, including the open air. The merits also include possible adaptation of the relevant devices to nonstandard examination conditions of heat exchange without loss of measurement accuracy [3].

The present work proposes a modified unsteady-state procedure for measuring the integral emissivity coefficient of the surface of heat-shielding materials in the vicinity of elevated temperatures, which can improve the measurement accuracy by taking into account the temperature field nonuniformity in a specimen [4].

\section{Modified unsteady-state procedure for emissivity measurement}

The modified unsteady-state method for measuring the integral emissivity coefficient is based on using a system of inverse problems of conductivity $[5,6]$. In this procedure, a cylindrical specimen of the heat-shielding material is preliminary heated to high temperature (no less than $500 \mathrm{~K}$ ), $T_{0}$, and the temperature is measured during cooling-down in a vacuumed chamber by a thermocouple installed inside the specimen. Two thermocouple measurement options are possible [4]:

- side surface and one of the end faces of the cylindrical specimen are heat insulated, and radiation emanates from the one of the end face surfaces (Fig. 1a);

- both of the end faces of the cylindrical specimen are heat insulated, and radiation emanates from the side surface (Fig. 2b).

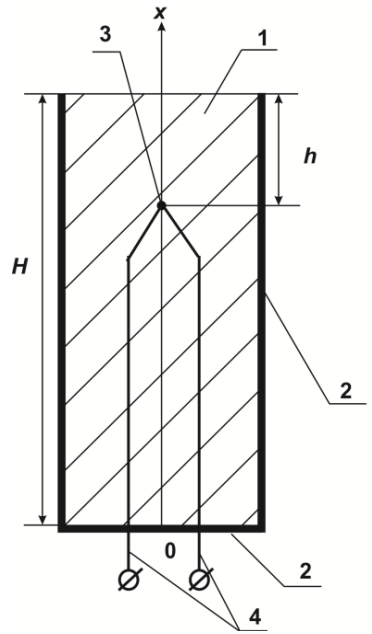

a)

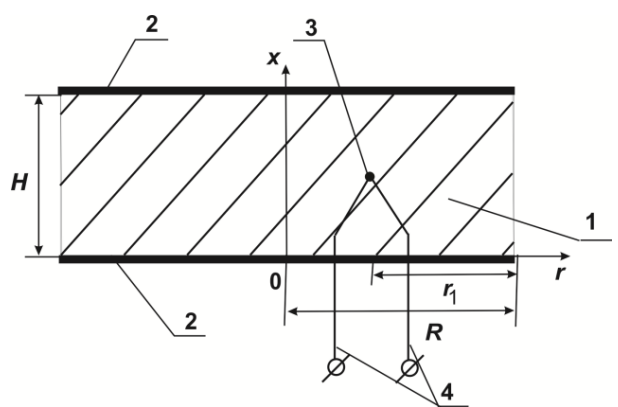

b)

Fig. 1. Emissivity coefficient measurement options: 1 - specimen, $2-$ heat insulator, $3-$ thermocouple junction, 4 - thermocouple leads.

In case the radiation emanates from one of the end face surfaces (Fig. 1a) when the specimen preheated to high temperature (at least $500 \mathrm{~K}$ ) is cooled down at cryogenic temperature (or at $T_{0}>>T_{c}, T_{0}$ - specimen temperature, $T_{c}$ - ambient temperature), the depth temperature is measured by the thermocouple installed at the symmetry axis of the specimen. With the assumption that the heat cross-flow along the radius of the cylindrical specimen is negligibly small and there is no convective heat exchange with the environment, the integral emissivity coefficient is determined from solving the inverse conductivity problem: 


$$
\begin{aligned}
& \rho c(T) \frac{\partial T(x, t)}{\partial t}=\frac{\partial}{\partial x}\left(\lambda(T(x, t)) \frac{\partial T(x, t)}{\partial x}\right), \\
& 0<x<H ; \quad 0<t \leq t_{k} ; \\
& T(x, 0)=T_{0} \equiv \text { const, } \\
& \quad 0 \leq x \leq H ; \\
& \frac{\partial T(0, t)}{\partial x}=0, \\
& \quad 0 \leq t \leq t_{k} ; \\
& \lambda(T(H, t)) \frac{\partial T(H, t)}{\partial x}=-\varepsilon \sigma T^{4}(H, t), \\
& \quad 0 \leq t \leq t_{k} .
\end{aligned}
$$

When the radiation is from the side surface of the cylindrical specimen (Fig. 1b), the integral emissivity is estimated from solving the inverse boundary problem for a twodimensional equation of heat conductivity:

$$
\begin{gathered}
\rho \cdot c(T(r, t)) \cdot \frac{\partial T(r, t)}{\partial t}=\frac{1}{r} \cdot \frac{\partial}{\partial r}\left(r \cdot \lambda(T(r, t)) \cdot \frac{\partial T(r, t)}{\partial r}\right), \\
0<r<R ; 0<t \leq t_{k} ; \\
T(r, 0)=T_{0} \equiv \text { const, } \\
0 \leq r \leq R \\
\frac{\partial T(0, t)}{\partial r}=0 \\
0 \leq t \leq t_{k} ; \\
\lambda(T(R, t)) \cdot \frac{\partial T(R, t)}{\partial r}=-\varepsilon \sigma T^{4}(R, t), \\
0 \leq t \leq t_{k} .
\end{gathered}
$$

The following notations are used in Figures 1-2 and problems (1)-(4) and (5)-(8): $\rho, c$, $\lambda$-density, specific heat capacity, heat conductivity coefficient of the specimen; $\varepsilon-$ integral emissivity coefficient; $T$-specimen temperature; $t$-time; $x, r$-cylindrical coordinates; $T_{0}$ - initial temperature of the emitting surface of the specimen; $H, R-$ specimen height and radius; $h, r_{1}$ - distance from the emitting surface to the thermocouple; $\sigma=5.6687 \cdot 10^{-8} \mathrm{~W} \cdot \mathrm{m}^{-2} \cdot \mathrm{K}^{-4}-$ Stefan-Boltzmann constant. 
The value of integral emissivity coefficient $\varepsilon$ in problems (1)-(4) and (5)-(8) is determined by minimizing the functional that includes a numerical solution to $T_{1}^{*}(t)$ and to the measured value of temperature $T_{1}(t)$ at the point where the thermocouple is installed:

$$
J(\varepsilon)=\sqrt{\frac{1}{t_{k}} \int_{0}^{t_{k}}\left[T_{1}^{*}(t)-T_{1}(t)\right]^{2} d t} .
$$

Problems (1)-(4) and (5)-(8) were solved numerically by using a variant of the Petukhov method [7] for the second-order boundary equation. The method has a fourthorder approximation in terms of spatial coordinate and a first order in terms of time. The computational algorithm allows simultaneously finding the sought-for function and its derivative. The difference equation system is solved by the matrix sweep method.

The application of the unsteady-state methods requires robust experimental data on specific heat capacity and heat conductivity of heat-shielding materials under examination. Instruments IT $-\lambda-400$ (Fig. 2, left) and IT $-c-400$ (Fig. 2, right) were employed to measure the heat conductivity coefficient and specific heat capacity as a function of temperature.

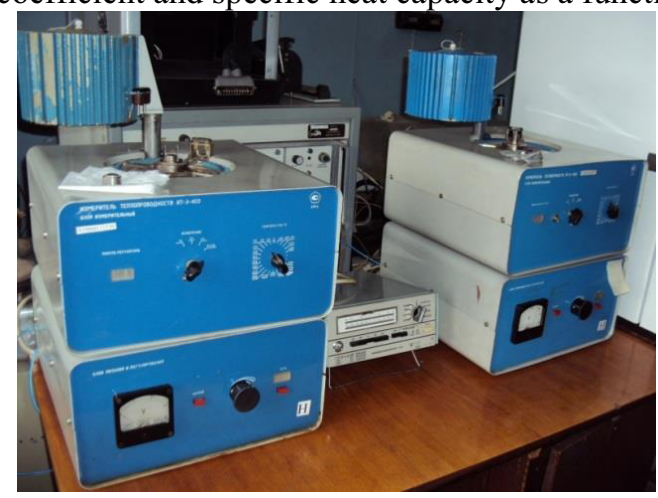

Fig. 2. Instruments for the measurement of thermophysical behaviors of materials.

\section{Results of computational experiments}

The two options of the suggested measurement procedure have been evaluated by solving a model problem. The computational experiment estimated the integral emissivity coefficient of the cylindrical specimen made of polished pyrolytic graphite during its cooling-down. The pyrolytic graphite had the following characteristics: density $\rho=2200 \mathrm{~kg} / \mathrm{m}^{3}$; heat conductivity coefficient $\lambda=2.8 \mathrm{~W} /(\mathrm{m} \cdot \mathrm{K})$; specific heat capacity $c=1340 \mathrm{~J} /(\mathrm{kg} \cdot \mathrm{K})$. The value of integral emissivity coefficient $\varepsilon$ of the polished pyrographite surface at $T=1200 \mathrm{~K}$ was 0.83 [8].

In the computational experiment with the radiation emanating from one of the end face surfaces, the cylindrical specimens had the following parameters: height $H=50 \mathrm{~mm}$, base radius $R=5 \mathrm{~mm}$, initial temperature $T_{0}=1000 \mathrm{~K} \equiv$ const. In the numerical experiments, the height $h$ from the emitting surface and the data spread of the thermocouple measurement of the depth temperature, $\Delta T=( \pm 2 \div \pm 10) \mathrm{K}$, were varied. Figure 3 illustrates the relative measurement error for integral emissivity coefficient $\delta \varepsilon$ plotted against $\Delta T$ and $h$. 


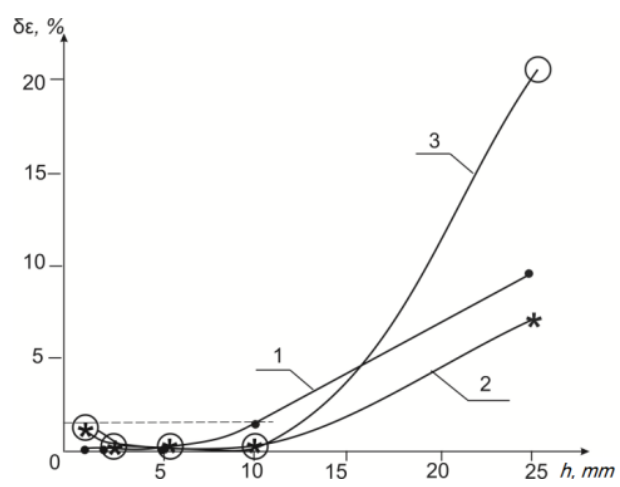

Fig. 3. Relative measurement error for integral emissivity coefficient $\delta \varepsilon$ plotted against the temperature measurement error: $1-\Delta T= \pm 2 \mathrm{~K} ; 2-\Delta T= \pm 5 \mathrm{~K} ; 3-\Delta T= \pm 10 \mathrm{~K}$.

The integral emissivity coefficient values subject to random perturbance of the measured temperature $\Delta T$ at fixed points and at different distance $h$ from the emitting surface are summarized in Table 1.

Table 1. Integral emissivity coefficient of pyrographite, $T_{0}=1000 \mathrm{~K}$.

\begin{tabular}{|c|c|c|c|c|c|c|c|c|c|c|}
\hline & \multicolumn{2}{|c|}{$h=1 \mathrm{~mm}$} & \multicolumn{2}{c|}{$h=2 \mathrm{~mm}$} & \multicolumn{2}{c|}{$h=5 \mathrm{~mm}$} & \multicolumn{2}{c|}{$h=10 \mathrm{~mm}$} & \multicolumn{2}{c|}{$h=25 \mathrm{~mm}$} \\
\hline$\Delta T, \mathrm{~K}$ & $\varepsilon$ & $\delta \varepsilon, \%$ & $\varepsilon$ & $\delta \varepsilon, \%$ & $\varepsilon$ & $\delta \varepsilon, \%$ & $\varepsilon$ & $\delta \varepsilon, \%$ & $\varepsilon$ & $\delta \varepsilon, \%$ \\
\hline \pm 2 & 0.834 & $<1$ & 0.835 & $<1$ & 0.832 & $<1$ & 0.84 & 1.2 & 0.91 & 9.6 \\
\hline \pm 5 & 0.82 & 1.2 & 0.835 & $<1$ & 0.832 & $<1$ & 0.835 & $<1$ & 0.89 & 7.2 \\
\hline \pm 10 & 0.82 & 1.2 & 0.832 & $<1$ & 0.833 & $<1$ & 0.81 & 1.2 & 1.00 & 20.5 \\
\hline
\end{tabular}

It can be seen from the results presented that when the temperature was measured over the distance range of $h \leq 10 \mathrm{~mm}$ from the emitting surface, the relative error was $\delta \varepsilon<1.5 \%$ (in Fig. 3, the error corridor is designated as a dashed line).

The computational experiment with the radiation coming from the side surface had the following conditions: cylindrical specimen height $H=10 \mathrm{~mm}$, base radius $R=10 \mathrm{MM}$, initial specimen temperature $T_{0}=1000 \mathrm{~K}$. The value of the thermocouple spatial coordinate varied, $\mathrm{r}_{1}=(1 ; 2 ; 5 ; 10) \mathrm{mm}$. The data scattering of the thermocouple temperature measurement varied in the range of $\Delta T( \pm 2 \div \pm 10) \mathrm{K}$.

Figure 4 displays the relative measurement error for integral emissivity coefficient $\delta \varepsilon$ plotted against $\Delta T$ and $h$. It is visible from the data in Figure 4 that when the temperature was measured over the distance range of $r_{l} \leq 5 \mathrm{~mm}$ from the emitting surface, the relative error was $\delta \varepsilon<1.5 \%$ (the error corridor is designated as a dashed line). The scatter in data from the thermocouple temperature measurement was $\Delta T=( \pm 2 \div \pm 10) \mathrm{K}$.

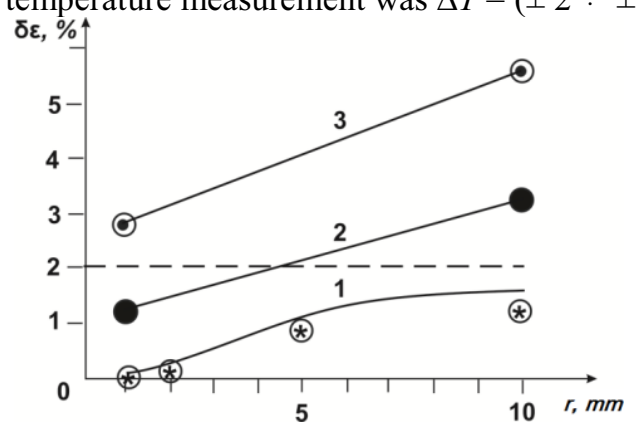

Fig. 4. Relative measurement error for integral emissivity coefficient $\delta \varepsilon$ plotted against the temperature measurement error: $1-\Delta T= \pm 2 \mathrm{~K} ; 2-\Delta T= \pm 5 \mathrm{~K} ; 3-\Delta T= \pm 10 \mathrm{~K}$. 
The numerical evaluation results of the modified unsteady-state method when the radiation emanates from the side surface of the cylindrical specimen have shown that the measurement accuracy for the integral emissivity coefficient of the surface of heat-shielding materials can be improved if the thermophysical experimental conditions comply with the following requirements:

- data scattering of the thermocouple temperature measurements should not be in excess of $\pm 5 \mathrm{~K}$;

- it is not advisable placing the informative thermocouple at the symmetry axis of the cylindrical specimen because, in this case, the measurement error for the integral emissivity coefficient may be as high as $3 \%$.

\section{Conclusion}

The findings from the computational experiments using the two temperature measurement options demonstrate that the modified unsteady-state procedure can improve the measurement accuracy for the integral emissivity coefficient of materials with low heat conductivity by taking the cognizance of the temperature field nonuniformity in the specimen.

This research carried out in 2015 was supported by "The Tomsk State University Academic D.I. Mendeleev Fund Program”, grant No 8.2.46.2015.

\section{References}

1. Yu.V. Polezhaev, F.B. Yurevich, Heat shield (Moscow, Energiya, 1976) [in Russian]

2. A.E. Sheindlin, Emitting properties of solid materials (Energiya, Moscow, 1974) [in Russian]

3. V.A. Arkhipov, V.D. Gol'din, I.K .Zharova, N.I. Kurilenko, G.Ya. Mamontov, Teplofizika i Aeromekhanika 19, 6 (2012) [in Russian]

4. V.A. Arkhipov, V.D. Gol'din, I.K. Zharova, N.I. Kurilenko, RU Patent 2468360, A method for measuring the integral emissivity coefficient of the surface of heat protection materials, Bulletin No. 33, 27.11.2012. [in Russian]

5. O.M. Alifanov, Inverse problems of heat exchange, (Mashinostoyeniye, Moscow 1988) [in Russian]

6. A.N. Tikhonov, V.D. Kal'ner, V.B. Glasko, Mathematical modeling of manufacturing processes and the inverse problem method in mechanical engineering (Mashinostoyeniye, Moscow, 1990) [in Russian]

7. V.D. Gol'din,, E.V. Yorkina, I.V., Issledovaniya po Ballistike i Smezhnym Voprosam Mekhaniki (Tomsk State University, Tomsk, 2001) [in Russian]

8. Yu.V. Polezhaev, A.A. Shishkov, Reference Book: Gas dynamic testing of heat shield (Promedek, Moscow, 1992) [in Russian] 